\title{
A interferência da sazonalidade sobre os grupos funcionais tróficos de invertebrados bentônicos presentes em substrato rochoso no Rio Palmital, União da Vitória - PR
}

\author{
Edina Costa Delonzek ${ }^{1 *}$ \\ Ana Carolina de Deus Bueno Krawczyk ${ }^{2}$ \\ ${ }^{1}$ Universidade Estadual do Centro-Oeste, Campus CEDETEG \\ Rua Simeão Varela de Sá, 3, Vila Carli, CEP 85040-080, Guarapuava - PR, Brasil \\ ${ }^{2}$ Departamento de Ciências Biológicas, Universidade Estadual do Paraná, Campus União da Vitória \\ Praça Coronel Amazonas, s/n, Caixa Postal 241, CEP 84600-000, União da Vitória - PR, Brasil \\ * Autor para correspondência \\ edinadelonzek@yahoo.com.br
}

Submetido em 03/09/2015

Aceito para publicação em 01/06/2016

\section{Resumo}

Este trabalho teve como objetivo conhecer os invertebrados bentônicos, bem como caracterizar os grupos funcionais tróficos (coletores, coletores-catadores, coletores-filtradores, fragmentadores, predadores e raspadores) encontrados em substrato rochoso em períodos sazonais no Rio Palmital, município de União da Vitória, Paraná, Brasil. As amostragens foram realizadas no período seco (junho/julho-2011) e chuvoso (novembro/ dezembro-2011). Os dados obtidos indicam que o grupo funcional dos coletores foi o mais frequente. Os resultados sugerem que há influências dos distúrbios provocados pela precipitação na fauna de invertebrados, durante o período de chuvas, sendo que determinadas espécies tróficas encontradas podem estar relacionadas com a presença de matéria orgânica como um recurso alimentar na dieta dos invertebrados aí presentes.

Palavras-chave: Ambientes lóticos; Guildas; Perturbações hidrológicas; Recurso alimentar

\section{Abstract}

Effect of seasonality on trophic functional groups of benthic invertebrates in rocky substrate in Rio Palmital, União da Vitória-PR. This study aimed to determine the benthic invertebrates found in the rocky substrate in seasonal periods in Rio Palmital, União da Vitória, Parana, Brazil, characterizing the trophic functional groups (collectors, collectors-gatherers, collectors-filterers, shredders, predators and scrapers). Sampling was carried out during the dry (June/July-2011) and rainy (November/December-2011) seasons. The data indicate that the functional group of collectors was the most frequent. The results suggest that there are influences of the disturbances caused by precipitation on the invertebrate fauna during the rainy season, and certain trophic species found may be related to the presence of organic matter as a food source in the diet of invertebrates present there.

Key words: Food resources; Guild; Hydrological disturbances; Lotic environments 


\section{Introdução}

A disponibilidade de alimento é um fator que controla a ocorrência e abundância de espécies, e esse é um recurso que faz com que a comunidade permaneça num determinado ambiente (SANTANA et al., 2010). Entretanto, poucos invertebrados de águas correntes são especializados em suas dietas (HYNES, 1970; PLAGUE et al., 1998). Estudos permitem realizar comparações da organização funcional de distintas comunidades, especialmente quando não são formadas por espécies comuns (CALLISTO; ESTEVES, 1998; SILVA et al., 2009).

A matéria orgânica presente em um sistema lótico é classificada de acordo com o tamanho que apresenta, sendo matéria orgânica particulada grossa (CPOM $>1$ $\mathrm{mm}$ ) composta de folhas vindas da vegetação marginal e macrófitas; matéria orgânica particulada fina (FPOM $0,5 \mathrm{~mm}<1 \mathrm{~mm}$ ) procedente da fragmentação de CPOM; perifiton, algas e microorganismos, e a matéria orgânica dissolvida (DOM $<0,5 \mathrm{~mm}$ ), encontrada em suspensão na superfície da água (CUMMINS, 1973; CALLADO, 2011).

Os invertebrados bentônicos podem ser classificados com relação ao seu comportamento alimentar, sendo agrupados nas seguintes categorias: coletores-catadores, que se alimentam de pequenas partículas de matéria orgânica por coleta nos depósitos de sedimento; coletores-filtradores, que capturam, por filtração, pequenas partículas de matéria orgânica em suspensão na coluna d'água; fragmentadores, que trituram folhas ou tecido vivo de planta vascular ou escavam madeira, participando da fragmentação e, indiretamente, da decomposição (FPOM); predadores, que engolem a presa inteira ou ingerem os fluidos do tecido corporal; e raspadores, que são adaptados a raspar superfícies duras, alimentam-se de algas, bactérias, fungos e matéria orgânica morta adsorvida aos substratos (MERRITT; CUMMINS, 1996).

Segundo Lampert e Sommer (2007), episódios naturais, como as perturbações hidrológicas, podem interferir nas cadeias tróficas dos invertebrados e entre os principais fatores físicos e químicos associados a esses eventos estão a temperatura e a velocidade de corrente, que representam mecanismos seletivos para a comunidade aquática, influenciando os processos fisiológicos e comportamentais, como o próprio estabelecimento no substrato. Além disso, as alterações causadas por fenômenos naturais causam perda de hábitat, reduzindo os recursos necessários para a sobrevivência da comunidade ali presente, podendo influenciar fortemente os padrões de distribuição espacial e temporal em comunidades bentônicas (BUSS et al., 2002; SILVEIRA; QUEIROZ, 2006).

Neste sentido, o objetivo deste estudo foi analisar a estrutura dos grupos funcionais tróficos da fauna de invertebrados bentônicos encontrados em substrato rochoso em períodos sazonais no Rio Palmital, União da Vitória-PR, Brasil.

\section{Material e Métodos}

\section{Área de estudo}

O estudo foi realizado na localidade do Palmital do Meio, no município de União da Vitória (Paraná, Brasil) (Figura 1), que está localizado no extremo sul do estado e pertence, em sua maioria, ao Terceiro Planalto Paranaense. Está inserido na Microrregião do Médio Iguaçu, na fronteira do estado de Santa Catarina, sendo limitado pela Serra da Esperança, com altitudes médias entre 700 e 900 m (MAACK, 2002).

FIGURA 1: Área de amostragem no Rio Palmital, na localidade do Palmital do Meio, União da Vitória - PR.

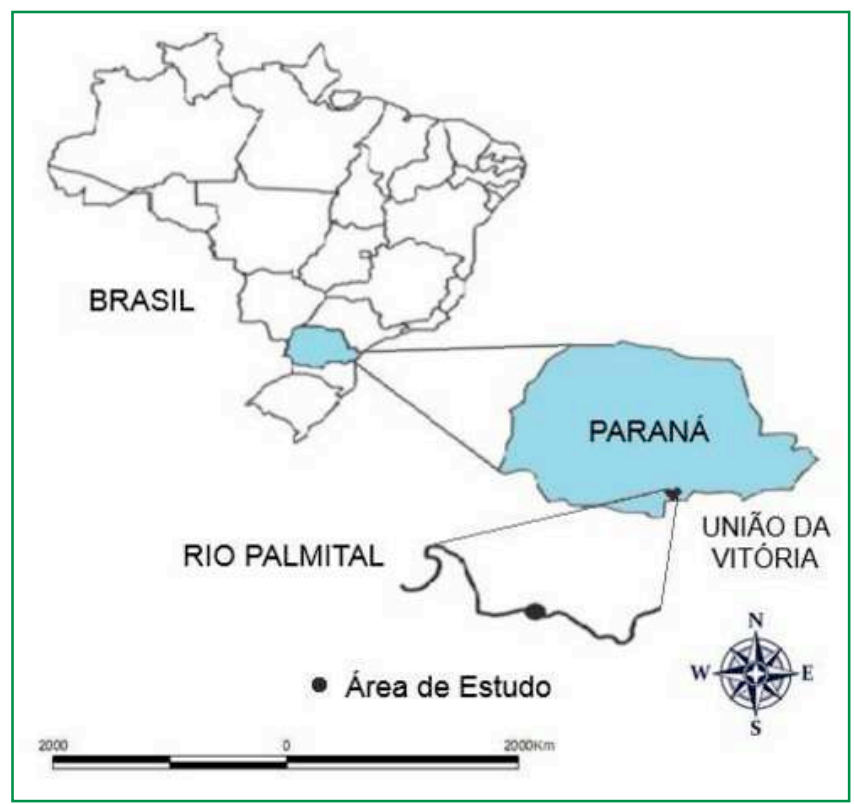


O Rio Palmital possui nascente na Serra da Esperança e é um afluente do rio Iguaçu na margem direita. As coletas ocorreram nos meses de maio e junho (2011) e novembro a dezembro (2011), por representarem, respectivamente, os períodos com menor e maior índice pluviométrico.

\section{Amostragem}

As coletas foram realizadas por meio de réplicas de substrato rochoso em áreas de corredeiras num trecho de $48 \mathrm{~m}$, com uma distância de $1,5 \mathrm{~m}$ entre uma réplica e outra, totalizando 60 unidades amostrais, sendo 30 para cada período. Cada réplica foi formada por um conjunto de nove pedras do rio com aproximadamente $2,8 \mathrm{~cm}$ de diâmetro, sendo que estas foram lavadas e ensacadas em tela de arame. Cinco réplicas foram retiradas semanalmente durante seis semanas de amostragem em cada período. O material foi fixado no campo com álcool a 70\%. No laboratório, os organismos foram visualizados em uma caixa de luz e identificados sob microscópio estereoscópico e microscópio óptico ao nível de gênero, exceto para a família Chironomidae, que foi identificada ao nível de subfamília (TRIVINHOSTRIXINO; STRIXINO, 1995; MERRIT; CUMMINS, 1996; MUGNAI et al., 2010). O cálculo da porcentagem de grupos funcionais tróficos foi baseado no número de indivíduos amostrados. De acordo com o modo de alimentação, os indivíduos foram agrupados e determinados para cada família de acordo com Merrit e Cummins (1996).

A temperatura foi medida em campo com o auxílio de um termômetro de coluna de mercúrio, enquanto os dados da vazão foram obtidos na estação da Fazenda Maracanã, na localidade do Palmital do Meio, União da Vitória - PR (COPEL, 2010).

\section{Resultados}

Os períodos amostrais foram significativamente diferentes em relação à temperatura da água $(\mathrm{p}=0,0000000095779)$, bem como em relação à vazão $(\mathrm{p}=0,001424)$. O período com maior precipitação apresentou maiores valores de temperatura $\left(20{ }^{\circ} \mathrm{C}\right) \mathrm{e}$ maior vazão $\left(7,28 \mathrm{~m}^{3} / \mathrm{s}\right)$, enquanto na estação com menor índice de precipitação a temperatura foi menor $\left(9^{\circ} \mathrm{C}\right) \mathrm{e}$ a vazão inferior $\left(2,44 \mathrm{~m}^{3} / \mathrm{s}\right)$.

A comunidade bentônica foi composta por 513 organismos, distribuídos em 21 taxa e cinco grupos tróficos funcionais. Desse total, 371 indivíduos foram coletados na estação seca e 142 na estação chuvosa.

O grupo trófico dos coletores $(n=182)$ foi o mais abundante no período da seca, principalmente pela presença de Simuliidae e Leptophlebiidade. O grupo de coletor/filtrador, composto pela subfamilia Ortocladiinae, foi representado por 101 organismos durante esse período. O grupo funcional dos raspadores ( $n=47)$ foi representado, na sua maioria, pela família Baetidae e os predadores $(n=32)$, principalmente por Gripopterygidae. O grupo de coletor/catador apareceu em menor quantidade $(n=9)$, com a ocorrência da subfamília Tanypodinae.

Durante a estação chuvosa, coletores foi o grupo trófico mais abundante $(\mathrm{n}=50)$, tendo sido representado por Caenidae, Simuliidae e Leptophlebiidae. Orthocladiinae $(n=43)$ foi o único representante do grupo coletor/filtrador encontrado neste período. A família Baetidae foi a representante do grupo dos raspadores $(\mathrm{n}=35)$. Em relação ao grupo dos predadores ( $n=14)$, Gripopterygidae, Perlidae e Libelulidae foram os que ocorreram neste período, e o grupo coletor/catador não ocorreu na estação chuvosa (Tabela 1). 
TABELA 1: Taxa, grupo trófico funcional (GTF), abundância total dos invertebrados bentônicos coletados nos períodos seco (junho/julho-2011) e chuvoso (novembro/dezembro-2011) no rio Palmital (União da Vitória - PR).

\begin{tabular}{rrrr}
\hline Taxa & GTF (MERRIT; CUMMINS, 1996) & Período Seco & Período Chuvoso \\
\hline
\end{tabular}

\section{Ephemeroptera}

\section{Baetidae}

Camelobaetidius sp.

$\begin{array}{lcc}\text { Raspador } & 28 & 19 \\ \text { Raspador } & 9 & 0 \\ \text { Raspador } & 8 & 14 \\ & & \\ \text { Coletor } & 0 & 1 \\ & & 3 \\ \text { Coletor } & 1 & 26 \\ \text { Coletor } & 6 & 15 \\ \text { Coletor } & 0 & \end{array}$

Tupiara sp.

Cloeodes sp.

Caenidae

Caenis sp.

Predador

Paragripoptterix sp.

Predador

1

5

Gripopteryx sp.

Predador

Perlidae

Macrogynoplax sp.

Predador

Predador

Anacroneuria sp.

Odonata

\section{Gomphidae}

Gomphoides sp.

Predador

Megapodagrionidae

Heteragrion sp.

Predador

\section{Libelulidae}

Zenithoptera sp.

Predador

Brechmorhoga sp.

Predador

\section{Coleoptera}

Psephenidae

Psephenus sp.

Raspador

Diptera

\section{Simuliidae}

Simullium sp.

Coletor

Chironomidae

Ortocladiinae

Tanypodinae

Chironominae

\begin{tabular}{ccc} 
Coletor-Filtrador & 101 & 43 \\
Predador & 8 & 0 \\
Coletor-Catador & 9 & 0 \\
\hline & $\mathbf{3 7 1}$ & $\mathbf{1 4 2}$
\end{tabular}


No Rio Palmital, foi observado o predomínio do grupo coletor seguido, em menor proporção, por coletores/filtradores, raspadores e predadores em ambos os períodos de coleta. O grupo coletor/catador foi representado apenas no período seco e em menor proporção que os demais (Figura 2).

FIGURA 2: Porcentagem de Grupos Funcionais Tróficos nos períodos chuvoso e seco no Rio Palmital, União da Vitória - PR.

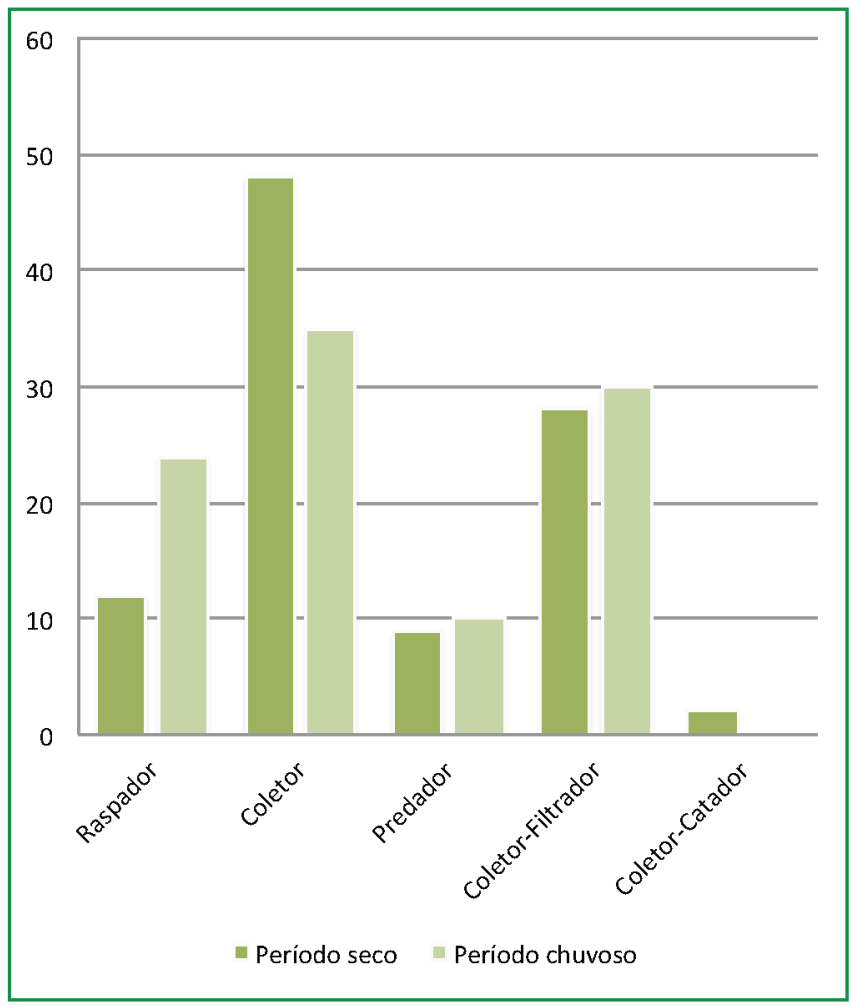

\section{Discussão}

No período chuvoso, houve maiores valores de temperatura, o que pode ter influenciado na redução dos invertebrados (FRANCO; LANDGRAF, 2002), uma vez que o aumento dessa variável propicia a diminuição da solubilidade do oxigênio (ANGELIER, 2003).

$\mathrm{Na}$ estação seca, houve maior abundância de invertebrados, o que pode estar relacionado à redução do volume de água, e, portanto, à menor vazão de água. Uma vazão menor favorece uma melhor estabilidade de hábitats e recursos alimentares para a colonização de invertebrados bentônicos. Fusari (2006) observou essa tendência no seu estudo, no qual a abundância total de organismos amostrados também foi superior no período mais seco do que no chuvoso. Durante a estação chuvosa, o crescente volume de água e a velocidade da correnteza possivelmente contribuíram para a remoção dos organismos do substrato, diminuindo o seu estabelecimento nos hábitats. Assim, os períodos sazonais interferem na estrutura das comunidades aquáticas e no seu recurso alimentar (REZENDE, 2007).

Uieda e Motta (2007) afirmam que as diferenças entre os dois períodos sazonais na composição dos grupos tróficos podem estar relacionadas com a estrutura das comunidades aquáticas, que é o resultado das interações bióticas, associadas com as variações ambientais em escalas espaciais e temporais.

Os cinco grupos funcionais encontrados neste estudo também foram observados por Copatti et al. (2010) num estudo realizado no Rio Cambará (Cruz Alta - RS). O grupo dos coletores foi o mais abundante em nosso estudo no período seco, um padrão similar ao encontrado por Callisto et al. (2001), num trabalho realizado na Serra do Cipó, na bacia do Rio São Francisco e do Rio Doce, que engloba uma série de córregos e rios, com características ecológicas ainda preservadas e águas de boa qualidade. As características locais do ambiente, de certa forma, também contribuem para o estabelecimento e comportamento das espécies (BUENO et al., 2003).

Desta forma, as diferenças encontradas entre o período seco e chuvoso na estrutura da fauna bentônica podem ser explicadas pela ação das chuvas torrenciais, que favorecem o aumento da vazão da água do rio, ocasionando uma desestruturação na comunidade local de invertebrados. Como as vazões afetam a disponibilidade de matéria orgânica para os invertebrados, aventa-se a hipótese de que a estrutura trófica analisada no Rio Palmital pode estar relacionada com a presença de matéria orgânica como um recurso alimentar na dieta dos invertebrados aí presentes.

\section{Referências}

ANGELIER, E. Ecology of streams and rivers. Boca Raton: Science Publishers, Inc., 2003. 215 p.

BUENO, A. A. P.; BOND-BUCKUP, G.; FERREIRA, B. D. P. Estrutura da comunidade de invertebrados bentônicos em dois 
cursos d'água do Rio Grande do Sul, Brasil. Revista Brasileira de Zoologia, Curitiba, v. 20, n. 1, p. 115-125, 2003.

BUSS, D. F.; BAPTISTA, D. F.; SILVEIRA, M. P.; NESSIMIAN, J. L.; DORWILLÉ, L. F. Influence of water chemistry and environmental degradation on macro invertebrate assemblages in a river basin in southest Brazil. Hydrobiologia, Brussels, v. 481, p. 125-136, 2002.

CALADO, S. C. M. Teia trófica dos macroinvertebrados em dois trechos do rio Sambaqui, Morretes - PR. 2011. 85 f. Dissertação (Mestrado em Ecologia e Conservação) - Universidade Federal do Paraná, Curitiba. 2011.

CAllisto, M.; ESTEVES, F. de A. Biomonitoramento da macrofauna bentônica de Chironomidae (Diptera) em dois igarapés amazônicos sob influência das atividades de uma mineração de bauxita. Oecologia Brasiliensis, Rio de Janeiro, v. 5, n. 1, p. 299309, 1998.

CALLISTO, M.; MORENO, P.; BARBOSA, F. A. R. Diversidade de habitats e grupos tróficos funcionais na Serra do Cipó, sudeste do Brasil. Revista Brasileira Biologia, São Carlos, v. 61, n. 2, p. 259-266, 2001.

COPATTI, C. E.; SCHIRMER, F.; MACHADO, J. V. Diversidade de macroinvertebrados bentônicos na avaliação da qualidade ambiental de uma microbacia no sul do Brasil. Perspectiva, Erechim, v. 34, p. 79-91, 2010.

COPEL - COMPANHIA PARANAENSE DE ENERGIA. Manual de acesso de geração distribuída. Norma técnica Copel 905100. 2010. Disponível em: <http://www.copel.com/hpcopel/normas/ ntcArquivos.nsf/0342A62F50C68EC4032577F500644B9A/ \$FILE/905100.pdf $>$. Acesso em: 04 fevereiro 2011.

CUMMINS, K. W. Trophic relations of aquatic insects. Annual Review of Entomology, Palo Alto, v. 18, p. 183-206, 1973.

FRANCO, B. D. G. M.; LANDGRAF, M. Microbiologia dos alimentos. São Paulo: Atheneu, 2002. 182 p.

FUSARI, L. M. Estudos das comunidades de macroinvertebrados bentônicos das Represas do Monjolinho e do Fazzari no campus da UFSCar, município de São Carlos, SP. 2006. 80 f. Dissertação (Mestrado em Ecologia e Recursos Naturais) - Universidade Federal de São Carlos, São Carlos. 2006.

HYNES, H. B. The ecology of running waters. Toronto: University of Toronto Press, 1970. 555 p.
LAMPERT, W.; SOMMER, U. Limnoecology - The ecology of lakes and streams. 2. ed. Oxford: Oxford University Press. 2007. $335 \mathrm{p}$.

MAACK, R. Geografia física do estado do Paraná. 3 ed. Curitiba: Imprensa Oficial. 2002. 350 p.

MERRIT, R. W.; CUMMINS, K. W. Uma introdução aos insetos aquáticos da América do Norte. 3. ed. Dubuque: Kendall/Caça Publishing Company, 1996. 722 p.

MUGNAI, R.; NESSIMIAN, J. L.; BAPTISTA, D. F. Manual de identificação de macroinvertebrados do estado do Rio de Janeiro. Rio de Janeiro: Technical Books, 2010. 176 p.

PLAGUE, G. R.; WALLACE J. B.; GRUBAUGH J. W. Linkages between trophic variability and distribution of Pteronarcys spp. (Plecoptera: Pteronarcyidae) along a stream continuum. American Midland Naturalist, Notre Dame, v. 139, p. 224-234, 1998.

REZENDE, C. F. Community structure of macroinvertebrates associated with leaf litter from riffles and pools from Central Amazon streams. Biota Neotropica, Campinas, v. 7, n. 2, p. 300$3005,2007$.

SANTANA, M. E. V.; SOUTO, L. S.; DANTAS, M. A. T. Diversidade de invertebrados cavernícolas na Toca da Raposa (Simão Dias - Sergipe): o papel do recurso alimentar e métodos de amostragem. Scientia Plena, São Cristóvão, v. 6, n. 12, p. 1-8, 2010 .

SILVA, F. L.; PAULETO, G. M.; TALAMONI, J. L. B.; RUIZ, S. S. Categorização trófica das comunidades de macroinvertebrados de dois reservatórios na região Centro-Oeste do estado de São Paulo, Brasil. Acta Scientiarum Biological Sciences, Maringá, v. 31, n. 1, p. 73-78, 2009.

SILVEIRA, M. P.; QUEIROZ, J. F. Uso de coletores com substrato artificial para monitoramento biológico de qualidade de água. Embrapa: Jaguariuna, 2006. 5 p. (Comunicado Técnico 39).

TRIVINHO-STRIXINO, S.; STRIXINO, G. Larvas de Chironomidae (Diptera) do estado de São Paulo. Guia de identificação e diagnose dos gêneros. São Carlos: Editora da Universidade de São Carlos, 1995. 229 p.

UIEDA, V. S.; MOTTA, R. L. Trophic organization and food web structure of southeastern Brazilian streams: a review. Acta Limnologica Brasiliensia, Botucatu v. 19, n.1, p.15-30, 2007. 